\title{
LA GESTIÓN DEL TIEMPO COMO COMPETENCIA DOCENTE EN LA CLASE DE DANZA CLÁSICA
}

\author{
A GESTÃO DO TEMPO COMO COMPETÊNCIA DOCENTE NA CLASSE DE \\ DANÇA CLÁSSICA
}

TIME MANAGEMENT AS A TEACHING COMPETENCE IN CLASSICAL DANCE LESSONS

\author{
José Carlos Fernández García*, Hayda García Molero*, Óscar Romero Ramos*
}

\begin{abstract}
Palabras clave: Docentes.

Música.

Tiempo.

Pruebas de

rendimiento.

Resumen: Esta investigación se ha llevado a cabo para conocer las diferencias en las variables temporales que conforman la competencia docente "Tiempo de compromiso motor" en la clase de Danza Clásica, bien acompañada de música grabada o por profesor pianista acompañante de danza. Se ha procedido al análisis de 50 grabaciones con ambos soportes musicales en un Conservatorio Superior de Danza de 89 alumnas que realizaban sus clases en un aula de ballet. Los resultados obtenidos destacan que según el soporte musical utilizado hay diferencias en las categorías de "Tiempo de Tarea" y "Tiempo de Organización", mostrándose un mayor valor en el "Tiempo de Tarea" en las sesiones en las que se utilizó la música en vivo, así como un menor tiempo en la "Organización", por lo que emplear la música en vivo podría proporcionar sesiones más productivas y con menor pérdida de tiempo.
\end{abstract}

Palavras chave: Docentes.

Música.

Tempo.

Provas de

rendimento.

Keywords:

Faculty.

Music.

Tempo.

Performance Tests.

Resumo: Esta pesquisa foi realizada com o objetivo de conhecer as diferenças nas variáveis temporais que compõem a competência docente, "Tempo de compromisso motor" nas aulas de Dança Clássica, seja acompanhado por música gravada ou por professor pianista acompanhando a dança. A análise de 50 gravações com ambos os suportes musicais foi realizada em um Conservatório Superior de Dança de 89 alunos que realizaram suas aulas em uma sala de ballet. Os resultados obtidos destacam que, de acordo com o suporte musical utilizado, existem diferenças nas categorias de "Tempo de Tarefa" e "Tempo de Organização", mostrando um maior valor no "Tempo de Tarefa" nas sessões em que a música ao vivo foi utilizada, bem como um menor tempo na "Organização", de modo que o uso de música ao vivo poderia proporcionar sessões mais produtivas com menor perda de tempo.

Abstract: This study looked into time variable differences in the teaching competence "Time of motor commitment" in Classical Dance lessons accompanied by either recorded music or a pianist teacher. Fifty recordings of 89 students of a Higher Education Dance Conservatory were analyzed in their ballet lessons with both musical supports. The results show differences in the categories of "Task Time" and "Organization Time" according to the musical support used, showing longer "Task Time" and shorter "Organization time" in lessons with live music. Therefore, live music could provide more productive lessons with less loss of time.
* Universidad de Málaga, Andalucía.

Málaga, España.

E-mail: jcfg@uma.es;

haydagmolero@gmail.com;

oromero@uma.es

Recebido em: 03-01-2019

Aprovado em: 03-06-2019

Publicado em: 27-12-2019

DOI

https://doi.org/10.22456/1982-8918.89401

(c) (i) () Licence 


\section{INTRODUCCIÓN}

Es necesario, para definir el marco contextual de este trabajo, mencionar la escasez de estudios que versan sobre la Danza Clásica y su acompañamiento musical en el ámbito educativo en los Conservatorios Superiores. Desde un punto de vista descriptivo y teórico, aparecen, de un tiempo a esta parte, trabajos académicos que abordan el acompañamiento pianístico de esta disciplina, y sobre la figura del profesor pianista acompañante en general. En este sentido, el trabajo de G-Molero y Fernández-García (2018) expone las investigaciones más recientes (CAVALLI, 2001; PAZ, DE 2006, 2011; LÓPEZ, 2008, 2009; AGUADO, 2009; SIRERA; SIRERA, 2009; WONG, 2011; FROSI, 2011; PUEYO, 2011, 2016; HERRERA; GÓMEZ, 2011; LAGUNA, 2013; PÉREZ-CHIRINOS, 2014; VALLÉS, 2015; TELLO, 2016).

En segundo lugar, un pilar básico en este estudio ha sido la vinculación y relación de la Danza Clásica con la Educación Física (DENDENA; CARMO, 2009), siendo evidente el componente cognitivo-motriz además del artístico dentro de la Danza Clásica (G-MOLERO; FERNÁNDEZ-GARCÍA, 2018). Partiendo de esta base, se hace necesario un acercamiento de ambas disciplinas, en la que una y otra se enriquezcan recíprocamente: la primera mediante la utilización de las distintas herramientas y técnicas de investigación ya empleadas en el área de la Educación Física y el deporte, con el consiguiente aumento de estudios, y la segunda, con un acercamiento a la Danza que enriquezca la formación de sus profesionales en el ámbito de la Expresión Corporal. Mediante este trabajo conjunto, el profesorado de ambas disciplinas se verá favorecido, de manera que el beneficiario último sea el alumnado.

Es manifiesta, entre los docentes, la dificultad de la impartición práctica de la Expresión Corporal en la enseñanza reglada (DENDENA; CARMO, 2009; MONTÁVEZ, 2011), incluyendo la Danza como manifestación incluida en la Expresión Corporal, cuyos futuros docentes de Educación Física consideran tener una formación muy escasa, lo que puede ser uno de los motivos que afecte a su dedicación e impartición (TROYA; CUÉLLAR, 2013).

Llegados a este punto, es importante destacar el objetivo (COTERÓN; SÁNCHEZ, 2010) según el cual, a pesar del lugar secundario que ha tenido el arte en general, y su manifestación a través del movimiento en concreto, es necesario aportar fundamentos teóricos y pautas de aplicación práctica para que esta realidad adquiera un carácter estable dentro del hecho educativo y de los valores sociales.

Por otra parte, un concepto que es considerado relevante en el ámbito educativo de la Educación Física es el Tiempo como dimensión y competencia docente que empezó a tener importancia con Carroll a partir de 1963, quien propuso un modelo referido al tiempo como variable más importante para explicar el aprendizaje escolar (FDEZ-REVELLÉS, 2008). Siguieron autores como Metzler (1979), con su tesis sobre la medida del Tiempo de aprendizaje en Educación Física; Siedentop, Birdwell y Metzler (1979), enfocados en medir la efectividad de la enseñanza en Educación Física; Metzler, DePaepe y Reif (1985), identificando futuras herramientas de observación temporal con su sistema electrónico Data Myte 801; Piéron (1986, 1988a, 1988b, 1999), de todos conocidos por sus aportaciones a la Didáctica y Pedagogía de la Educación Física y su interés por la eficacia de la enseñanza de ésta; más recientemente, Lozano y Viciana (2003), estudiando la competencia de gestión del Tiempo y su incidencia en la organización de la clase; y Fdez-Revellés (2003), que con su Tesis doctoral sobre "La Competencia Docente Tiempo en EF" ha seguido ampliando y abriendo camino en este campo de investigación. 
En estos trabajos sobre el Tiempo, se hace evidente el hecho de la pérdida o reducción del "Tiempo de programa" asignado a una sesión de Educación Física, así, desde el Tiempo Total que tiene en el programa una clase normal de EF, hasta el Tiempo real que se dedica, hay una reducción de tiempo $($ OLMEDO, 2000) existiendo estudios donde se comprueba que el Tiempo de práctica no representa más de un 30\% del Tiempo Útil (PIÉRON, 1992; OLMEDO, 2000).

Son pocas las investigaciones específicas en Danza en este sentido, pero ¿qué ocurrirá en las clases de Danza Clásica cuyo componente cognitivo motriz también es innegable? Bien es cierto que la clase de Danza Clásica posee un orden previo establecido, pero se comprueban aspectos comunes a las sesiones de EF como son la colocación/retirada de barras para la práctica, la espera de turno de práctica para variaciones grupales o incluso imprevistos en el desarrollo de la sesión. Estudiando este concepto y los factores que interfieren en él, se podría conseguir un resultado más satisfactorio de todo el proceso de enseñanza-aprendizaje. Dentro de esta metodología, hay un componente importante en la clase de Danza Clásica que no se da en la Educación Física, y es la figura del pianista acompañante de danza que debe trabajar en común y en correcto acuerdo con el profesorado encargado de estas enseñanzas. No obstante, no siempre es posible contar con pianista acompañante de danza en todas las clases de los centros educativos (sobre todo en las Enseñanzas Básicas), pero sí siguen siendo requeridos por los docentes de los Conservatorio Elementales, Profesionales y Superiores de Danza, como centros públicos de enseñanza, así como, también, en otros ambientes privados de estas especialidades como son Compañías de Danza y algunas escuelas privadas. Estos profesionales, a pesar de recurrir a la música grabada como solución urgente, prefieren al pianista de danza como artífice para unir técnica y arte en la clase de ballet (PÉREZ, 2002; G-MOLERO; ROMERO-RAMOS, 2016).

De esta manera, dentro del objetivo principal de esta investigación aparece el estudio del papel desempeñado por el profesor pianista acompañante de danza y su aportación a la productividad de la clase de Danza Clásica. Con los resultados obtenidos se podría ayudar a configurar una nueva realidad educativa, de manera que aporten soluciones, conocimiento y reconocimiento a la especificidad y trabajo del pianista de danza.

\section{MATERIALES Y MÉTODO}

\subsection{MUESTRA}

La muestra estuvo compuesta por 89 alumnas de danza, pertenecientes a los cursos $1^{\circ}$ a $4^{\circ}$ de Enseñanzas Superiores oficiales de un Conservatorio Superior de Danza, de los estilos de Danza Clásica, Danza Contemporánea, Danza Española y Baile Flamenco. Se hace necesario aclarar que las clases de técnica de Danza Clásica, de las que se han realizado las 50 grabaciones de esta investigación, está presente como contenido curricular en los cuatro estilos de enseñanza mencionados, no sólo en Clásico, sino también en Contemporáneo, Español y Flamenco. Tras estos cuatro años de estudios superiores (cursados después de superar cuatro cursos de Enseñanzas Básicas y seis de Enseñanzas Profesionales) el alumno obtendrá el Título superior de danza, en la especialidad cursada: Coreografía e Interpretación o Pedagogía de la Danza, siendo equivalente, a todos los efectos, al grado universitario. Además, podrá seguir formándose hacia unos estudios más especializados en las enseñanzas de Máster y Estudios de Doctorado. Estos estudios permitirán acceder a profesiones como 
coreógrafos, intérpretes o maestros de baile flamenco, danza clásica, danza española o danza contemporánea según la especialidad y estilo que se haya cursado.

Han participado como docentes: cinco profesoras y dos profesores de Danza Clásica, una profesora pianista acompañante y cuatro profesores pianistas acompañantes.

Con anterioridad al estudio, se cumplieron con los principios de ética reflejados en la declaración de Helsinky de 1964, revisados en 2013, la cual define las pautas éticas para la investigación en seres humanos, para lo que se obtuvo el consentimiento de los participantes, igualmente durante todo el proceso de investigación y posteriormente se actuó bajo lo dispuesto en la ley orgánica 15/1999, de 13 de diciembre, de protección de datos de carácter personal.

\subsection{INSTRUMENTO}

Se ha empleado una estrategia de investigación pre-experimental, con un diseño intragrupo y en un contexto natural (CAMPOS; GARRIDO; CASTAÑEDA, 2011).

El instrumento para el proceso de recogida de datos ha sido la grabación en vídeo en formato.mpg con una cámara Sony modelo DCR-SR37 HDD. De las técnicas de observación sistemática, se ha utilizado el registro de duración temporal. Los datos derivados del registro de duración se expresan en minutos y segundos, pasándolos directamente a cifras de porcentaje.

Para la recogida y tratamiento de los datos se ha empleado el software Match Vision Studio Premium (MOTS). Como afirman Hernández, Castellano, Camerino, Jonsson, Villaseñor, Lopes y Anguer (2014), desde que este programa fue propuesto por Castellano, Perea y Alday (2005), y traducido al inglés (CASTELLANO; PEREA; ALDAY; HERNÁNDEZ-MENDO, 2008) con la nomenclatura de MOTS (Measuring and Observation Tool in Sports) hasta hoy, muchos trabajos lo han empleado en el ámbito deportivo: fútbol (CAMERINO; CHAVERRI; ANGUERA; JONSSON, 2012; CASAMICHANA; CASTELLANO, 2010; GARCÍA; RIAL; REAL, 2012; LOSADA, 2012), baloncesto (FERNÁNDEZ; CAMERINO; ANGUERA; JONSSON, 2009), voleibol (HERNÁNDEZ-MENDO; MONTORO; REINA; FERNÁNDEZ-GARCÍA, 2012), balonmano (LOZANO; CAMERINO, 2012), o judo (GUTIÉRREZ-SANTIAGO; PRIETO; CAMERINO; ANGUERA, 2013; PRIETO; GUTIÉRREZ-SANTIAGO; OLEGUER; ANGUERA, 2013) y, también, en otros ámbitos relacionados (FERNÁNDEZ; SÁNCHEZ; JIMÉNEZ; NAVARRO; ANGUERA, 2012; LEWIS; D'ANDREA; ROSENBLUM, 2012; NEUMUTH; KASCHEK; NEUMUTH; CESCHIA; MEIXENSBERGER; STRAUSS; BURGERT, 2012).

De entre los distintos instrumentos que se ocupan del registro de variables temporales en el ámbito educativo de la Educación Física (LOZANO; VICIANA; PIÉRON, 2006; RAMÍREZ; LOZANO; SAN-MATÍAS; ZABALA; VICIANA, 2006), y, dada la falta de trabajos en el ámbito de la Danza Clásica, se ha escogido para esta investigación, la herramienta observacional de Telama, Varstala, Heikinaro-Johansson, y Paukku (1986), cuyas variables temporales pueden extrapolarse a la realidad educativa de la clase de Danza Clásica. Estas seis categorías que registran el comportamiento del alumnado son:

a. Organización, organizing (ORG)

b. Siguiendo las explicaciones del profesor sobre la materia de clase, following teaching (EXP)

c. Recibiendo feedback, getting feedback (RFD)

d. Tiempo de práctica de la tarea, time on task (TTA)

e. Esperando turno para prácticar, waiting for turn (TUR)

f. Otras actividades, other activities (OTA) 


\subsection{PROCEDIMIENTO}

Se registraron 50 clases de técnica de Danza Clásica, impartidas todas ellas en un Conservatorio Superior de Danza, con un Tiempo Programado o tiempo definido por los organismos públicos en los horarios escolares (FDEZ-REVELLÉS, 2008), de hora y media de duración.

Concluido el trabajo de campo, se procedió al análisis de la información mediante el software MOTS. Esto requirió la configuración de la herramienta observacional y la recogida de todos los datos en el módulo correspondiente, donde se registraron las distintas categorías observadas, tanto individualmente, como simultáneamente con otra categoría, en la línea temporal. Las seis variables utilizadas para este estudio han sido registradas con sus correspondientes valores en la línea temporal de cada sesión.

Los parámetros que recogen las distintas categorías son:

a. Organización: Tiempos, en minutos y segundos, que se dedican a la preparación del material para el adecuado discurrir de la sesión. Incluye la preparación del equipo de sonido, colocación o retirada de barras, y también los desplazamientos hacia la fuente de sonido, tanto para iniciar, cambiar o interrumpir la música, como para dirigirse al pianista. Comienza en el momento en que el profesor se dirige a la fuente de sonido (música o piano) a preparar la música (ya sea al inicio de la clase o durante ésta) o bien cuando éste y algunas alumnas van hacia las barras para colocarlas o retirarlas. También se ha acordado registrar como Organización el breve tiempo que a veces el profesorado dedica a controlar la asistencia de las alumnas dado que es un tiempo para organizar al alumnado y comenzar o proseguir la clase. A su vez, se ha observado cómo el profesor, individual o conjuntamente con las alumnas, debe parar a escuchar la música previamente a la actividad, lo que también se ha enmarcado en esta categoría dado que es una acción necesaria para la ejecución de la tarea. Esta categoría finaliza en el momento que se observa el inicio de otra actividad (Explicación, Tiempo de Tarea, etc.).

b. Siguiendo las explicaciones del profesor sobre la materia de la clase: El profesor o profesora "marca" la variación coreográfica. Hay que distinguir esta categoría de la siguiente, "recibiendo feedback", que se refiere a las correcciones que reciben las alumnas, tanto de manera individual como grupal. Se ha observado cómo esta explicación se realiza previamente a la ejecución del ejercicio y no siempre de la misma forma: con números ("conteo"), nombre de los pasos, movimientos de su propio cuerpo, o, incluso, pidiendo a una alumna la realización de los mismos ante las compañeras. Esta categoría comienza con el primer gesto, movimiento, o palabra del profesor o profesora y finaliza con la siguiente actividad que, normalmente será, por lo observado, el tiempo dedicado a preparar la música correspondiente o dirigirse al pianista según el caso (Organización) para seguidamente iniciar la variación coreográfica (Tiempo de Tarea). Es necesario aclarar también que alguna indicación sobre la calidad o ejecución del movimiento de manera general, y antes de realizar la variación no se ha considerado como Feedback sino un tiempo perteneciente a la Explicación.

c. Recibiendo Feedback: Recoge todas las correcciones del profesor o profesora tanto de manera individualizada como grupal. Empieza desde que el profesorado se dirige corporal o verbalmente a una alumna o grupo de alumnas hasta que deja de hacerlo.

d. Tiempo de práctica de la tarea: Tiempo empleado en la ejecución de la variación, comienza desde el inicio de la primera nota musical, hasta la conclusión del último movimiento o 
posición fija y llegada a la relajación muscular, que deberá ser, normalmente, sincronizada en todo el alumnado. Esta categoría recoge la ejecución "formal", con la implicación de la musculatura necesaria y atención de la alumna, y no el "marcaje" del ejercicio que se suele realizar durante la Explicación para memorizar la concatenación de los pasos de una manera más interiorizada.

e. Esperando turno para practicar: Esta categoría se ha registrado de manera simultánea al Tiempo de Tarea, dado que se produce en las variaciones de "centro" (CAVALLI, 2001), donde se realizan ejercicios por grupos de 2 ó 3 (diagonales, por ejemplo) ó 5 ó 6 (pequeños saltos, etc.) alumnas. Empieza desde que las alumnas están colocadas por grupos o parejas preparadas para iniciar la actividad y termina en el momento que el último grupo o pareja comienza su turno de práctica.

f. Otras actividades: Tanto imprevistos o interrupciones, como calentamientos previos, estiramientos finales y cualquier otra actividad que no se corresponda con Explicación, Tiempo de Tarea, o Recibiendo Feedback, incluyendo conversaciones o consejos sobre lesiones o casos ocasionales. Se empieza a registrar desde el primer segundo que comienza la actividad, ya sea una llamada a la puerta del aula por una interrupción externa, estiramientos 0 calentamientos individuales del alumnado y finaliza con el inicio o continuación de la siguiente actividad (Tiempo de Tarea, Explicación, etc.). Se aclara aquí que cuando el ejercicio de estiramiento o relajación sea de todo el alumnado y guiado por el profesor o profesora será registrado como Tiempo de Tarea. No así cuando existan en el aula una o más alumnas que de forma individual estén calentando o estirando (Otras Actividades), lo que podrá ocurrir simultáneamente a otras actividades como Organización (en el caso de que el profesor esté preparando el aparato de música, otras compañeras colocando las barras, etc.).

Una vez terminado el período de grabaciones se obtuvieron todos los registros, con los que se realizó un análisis de las frecuencias con duraciones en minutos y segundos y un análisis en una tabla de contingencia $n$-dimensional. Todos estos registros fueron integrados en una matriz, junto a datos como: el soporte musical de la sesión (música grabada o pianista de danza), curso, estilo (Clásico, Contemporáneo, Español o Flamenco), género del docente, experiencia del profesor o profesora, etc. Finalmente, el programa estadístico utilizado para el tratamiento de los datos fue SPSS Statistics.

Figura 1- Captura de pantalla del programa MOTS en la opción de "recogida de datos".

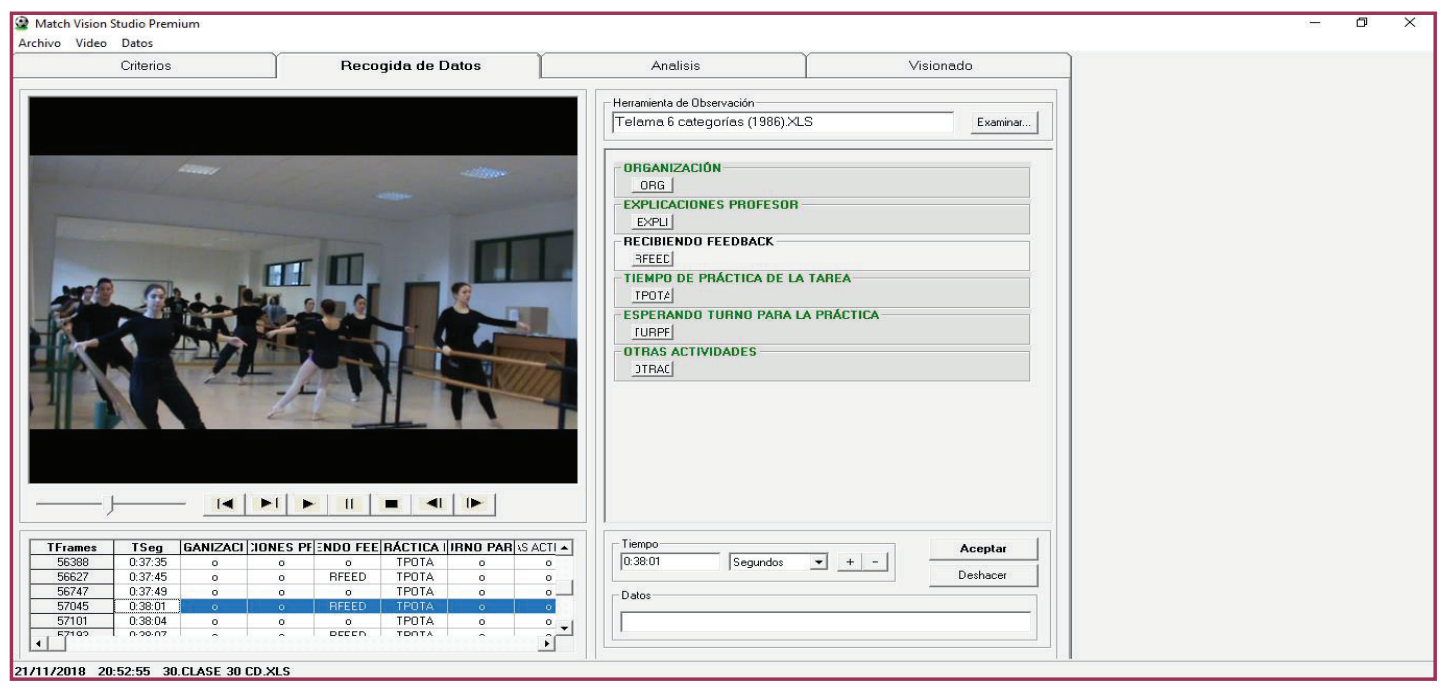

Fuente: De los autores 


\section{RESULTADOS}

Se expondrán, a continuación, los resultados obtenidos de la muestra investigada según el soporte musical empleado, dividiéndola en dos grupos: muestra con música grabada (23 clases) y muestra con pianista de danza (27 clases).

Para el estudio de la normalidad de los datos se ha aplicado el test de KolmogorovSmirnov, presentando un comportamiento normal la distribución de la muestra ya que todas las variables analizadas dan valores no significativos.

En la tabla 1 se exponen los estadísticos descriptivos y de contraste entre las dos muestras de estudio.

Tabla 1 - Estadísticos descriptivos y de contraste entre las dos muestras de estudio (música grabada: $C D$ ( $n=23$ ) y pianista de danza: $P D(n=27))$

\begin{tabular}{|c|c|c|c|}
\hline & Música & Media \pm Desv. típ. & $p$ \\
\hline \multirow{2}{*}{ Frecuencia Organización (Sucesos) } & $C D$ & $1131,26 \pm 31,28$ & \multirow{2}{*}{, $000^{*}$} \\
\hline & PD & $226,81 \pm 8,45$ & \\
\hline \multirow{2}{*}{ Tiempo Organización (Minutos y segundos) } & $C D$ & $00: 17: 07 \pm 0: 04: 37$ & \multirow{2}{*}{, $000^{*}$} \\
\hline & PD & $00: 02: 54 \pm 0: 01: 58$ & \\
\hline \multirow{2}{*}{ Frecuencia Explicación (Sucesos) } & $C D$ & $441,04 \pm 10,53$ & \multirow{2}{*}{, $000^{*}$} \\
\hline & PD & $221,67 \pm 5,67$ & \\
\hline \multirow{2}{*}{ Tiempo Explicación (Minutos y segundos) } & $C D$ & $00: 20: 27 \pm 0: 04: 39$ & \multirow{2}{*}{, $006^{\star}$} \\
\hline & PD & 00:24:04 $\pm 0: 04: 08$ & \\
\hline \multirow{2}{*}{ Frecuencia Recibiendo Feedback (Sucesos) } & $C D$ & $1134,52 \pm 48,35$ & \multirow{2}{*}{,401 } \\
\hline & PD & $1123,96 \pm 39,77$ & \\
\hline \multirow{2}{*}{ Tiempo Recibiendo Feedback (Minutos y segundos) } & $C D$ & $00: 33: 27 \pm 0: 15: 13$ & \multirow{2}{*}{,708 } \\
\hline & PD & $00: 32: 01 \pm 0: 11: 33$ & \\
\hline \multirow{2}{*}{ Frecuencia Tiempo de Tarea (Sucesos) } & $C D$ & $1190,78 \pm 118,44$ & \multirow{2}{*}{,380 } \\
\hline & PD & $2215,70 \pm 79,05$ & \\
\hline \multirow{2}{*}{ Tiempo Tiempo de Tarea (Minutos y segundos) } & $C D$ & $00: 28: 36 \pm 0: 05: 02$ & \multirow{2}{*}{, $026^{\star}$} \\
\hline & PD & $00: 32: 21 \pm 0: 06: 15$ & \\
\hline \multirow{2}{*}{ Frecuencia Turno esperando para la práctica (Sucesos) } & $C D$ & $334,22 \pm 54,36$ & \multirow{2}{*}{154} \\
\hline & PD & $557,96 \pm 60,51$ & \\
\hline \multirow{2}{*}{ Tiempo Turno esperando para la práctica (Minutos y segundos) } & $C D$ & $00: 03: 55 \pm 0: 04: 58$ & \multirow{2}{*}{,071 } \\
\hline & PD & 00:06:57 $\pm 0: 06: 22$ & \\
\hline \multirow{2}{*}{ Frecuencia Otras Actividades (Sucesos) } & $C D$ & $99,57 \pm 9,74$ & \multirow{2}{*}{, $021^{*}$} \\
\hline & PD & $44,96 \pm 2,17$ & \\
\hline \multirow{2}{*}{ Tiempo Otras Actividades (Minutos y segundos) } & $C D$ & $00: 07: 46 \pm 0: 06: 24$ & \multirow{2}{*}{,801 } \\
\hline & PD & 00:08:08 $\pm 0: 03: 35$ & \\
\hline
\end{tabular}

Fuente: Datos de los autores *nivel de significación $p<0,05$

\subsection{ORGANIZACIÓN}

Con respecto a la variable temporal, Organización, en ambas muestras se han obtenido resultados con diferencias estadísticamente significativas, tanto en la frecuencia $(131,26 \pm 31,28$ 
sucesos con música grabada y $26,81 \pm 8,45$ sucesos con pianista de danza), como en el tiempo empleado (17'07" \pm 4 '37" con música grabada y 2'54" \pm 1 '58" con pianista de danza). Esta categoría refleja el número de veces que el profesorado realiza desplazamientos a la fuente de sonido en el aula, ya sea ésta el reproductor de música o el profesor pianista acompañante, además de los comentados tiempos requeridos para preparación del material necesario para el correcto desarrollo de la clase, tales como colocación o retirada de barras, etc. Esta gran diferencia de valores obtenida se ha manifestado muy relacionada con la mayor adecuación de la música proporcionada por el pianista de danza a los movimientos coreográficos expuestos y requeridos por el profesor, lo que no siempre ocurre con la música grabada, momento en el que es necesario escuchar y cambiar la música elegida.

En relación al tiempo empleado en Organización se han registrado también tiempos muy diferentes: $17^{\prime} 077^{\prime \prime} \pm 04^{\prime} 37^{\prime \prime}$ en la muestra con música grabada, frente a: $02{ }^{\prime} 54^{\prime \prime} \pm 01^{\prime} 58^{\prime \prime}$ con el pianista de danza, resultando que hay diferencias estadísticamente significativas entre ambas muestras.

Figura 2 - Frecuencia Organización (izquierda) y Tiempo Organización (derecha).

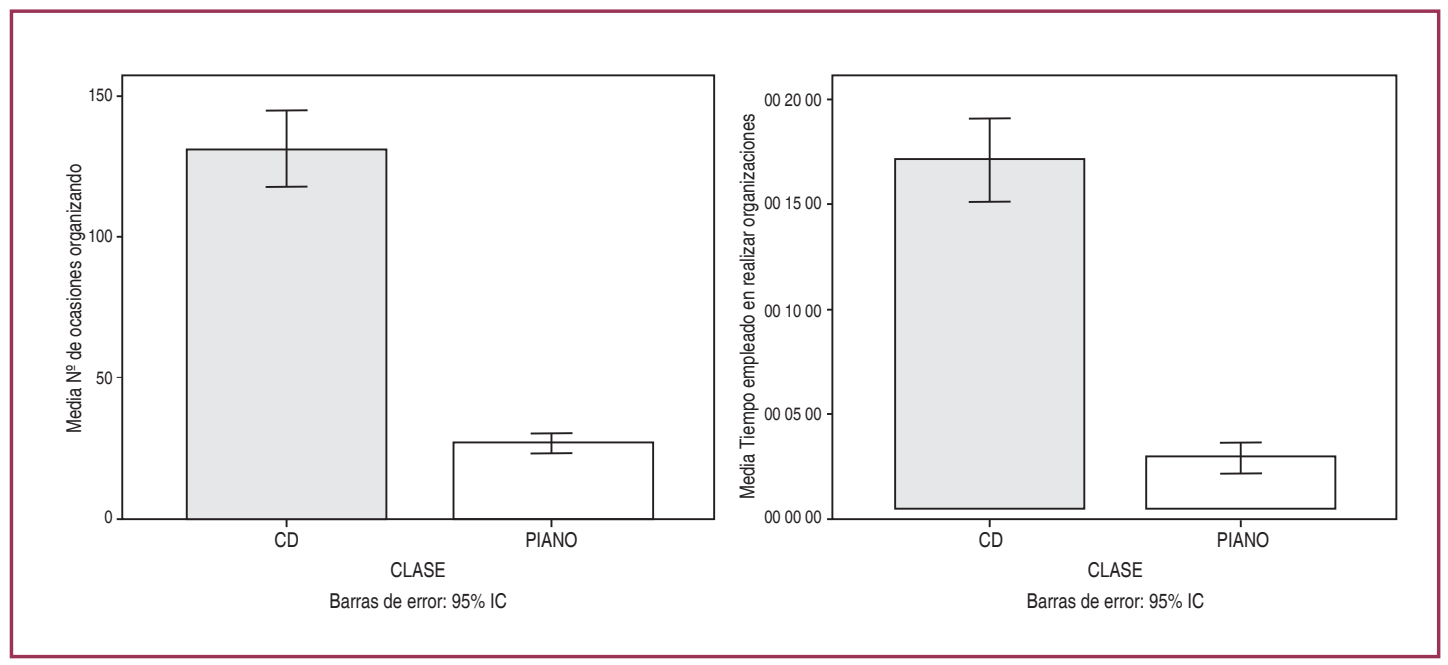

Fuente: Datos de los autores

\subsection{SIGUIENDO LAS EXPLICACIONES DEL PROFESOR}

Esta es una categoría en la que también se han registrados diferencias con respecto a ambas muestras. En las sesiones con música grabada prácticamente se duplican el número de ocasiones en las que el profesorado debe marcar la variación coreográfica: $41,04 \pm 10,53$ vs $21,67 \pm 5,67$ de media con el pianista de danza. Esto puede estar relacionado con las necesarias "pruebas" que se requieren con la música grabada y con una mayor necesidad de escucha de la música grabada por el profesorado y alumnado, en comparación con la música en vivo. En cuanto al tiempo total de Explicación hay una mayor franja de valores en el caso del pianista de danza: 24 '04" \pm 04 '08" vs $20 ’ 27$ " \pm 4 '39" de la música grabada. Se aprecia, pues, mayor tiempo en el que el profesorado marca la variación para la muestra con piano, pero menor frecuencia en relación con las clases con música grabada. 
Figura 3 - Frecuencia Explicación (izquierda) y Tiempo Explicación (derecha).
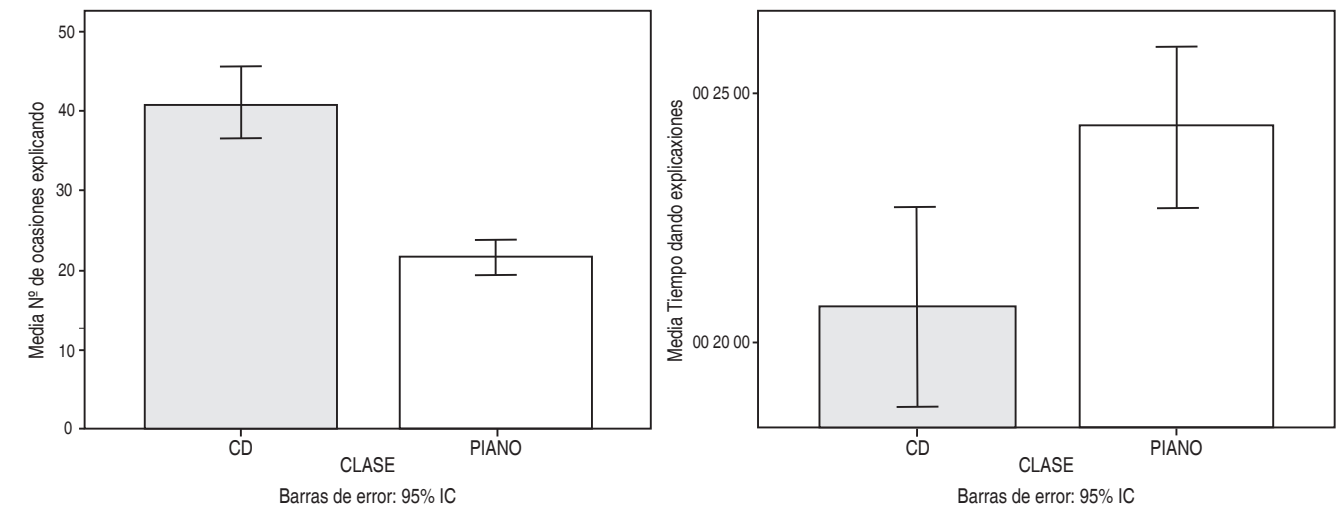

Fuente: Datos de los autores

\subsection{RECIBIENDO FEEDBACK}

Esta variable ha sido registrada simultáneamente a la de Tiempo de Tarea y no solo al finalizar la ejecución. Además, se ha presentado tanto individualmente, como grupalmente. En esta categoría no se han recogido grandes diferencias entre ambas muestras, sí se refleja una pequeña reducción en el número de ocasiones que el alumnado ha recibido feedback en el caso de las clases acompañadas con el pianista de danza. Los tiempos son 33'27" $\pm 15^{\prime} 13^{\prime \prime}$ para música grabada y $322^{\prime} 01{ }^{\prime \prime} \pm 11$ '33" en las clases con pianista de danza. De modo que el tiempo que dedica el profesorado a indicaciones a las alumnas no parece verse afectado por el soporte musical, no obstante, esa ligera reducción en el caso de las sesiones con el soporte del pianista de danza pudiera reflejar una mayor comprensión de la dialéctica musical por parte del alumnado.

Figura 4 - Frecuencia Recibiendo Feedback (izquierda) y Tiempo Recibiendo Feedback (derecha).
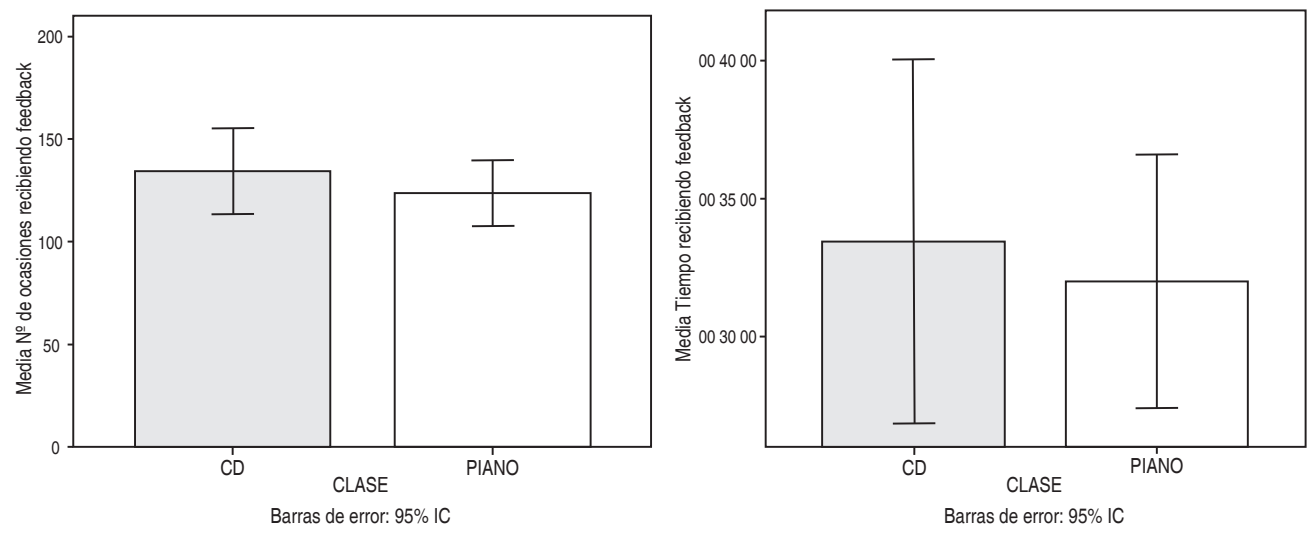

Fuente: Dados de los autores

\subsection{TIEMPO DE PRÁCTICA DE LA TAREA}

Con respecto al Tiempo de Tarea o tiempo específico dedicado a la ejecución de las distintas variaciones coreográficas propuestas por el profesor, vuelven a aparecer diferencias 
significativas entre ambas muestras. En ambos parámetros, frecuencia y tiempo, se registraron valores mayores en la muestra con pianista de danza frente a la muestra con música grabada, de modo que un mayor Tiempo de Tarea podría llevar aparejado mayor aprovechamiento de la sesión por parte del alumnado, así lo indican por un lado Fdez-Revellés (2008) en el que un registro de Tiempo útil elevado durante la sesión implica generalmente progresos a nivel físico y de aprendizaje motor. También Siedentop, (1999), citado por Fdez-Revellés (2008) afirma que el aprendizaje varía considerablemente según las actividades practicadas, siendo las de acondicionamiento físico y de danza las que producen tasas más elevadas.

Figura 5 - Frecuencia Tiempo de Tarea (izquierda) y Tiempo Tiempo de Tarea (derecha).

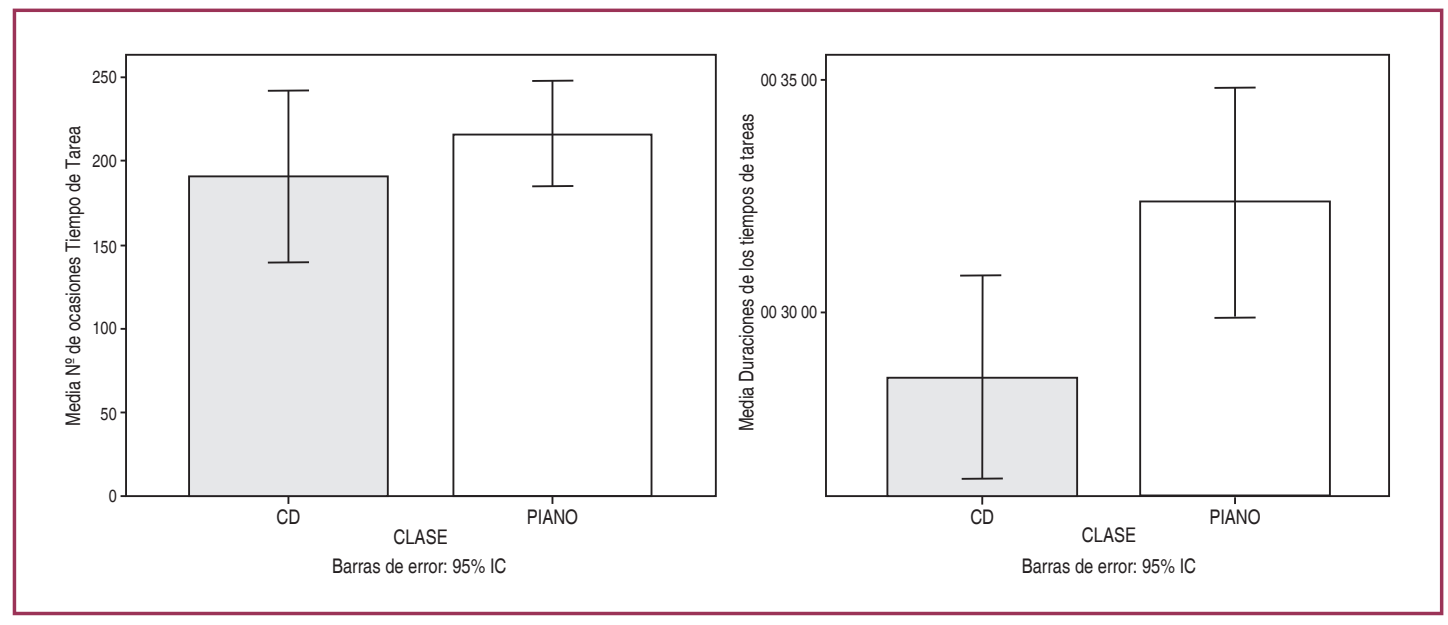

Fuente: Datos de los autores

\subsection{ESPERANDO TURNO PARA PRACTICAR}

No se han registrado diferencias estadísticamente significativas en esta variable temporal. En ambos parámetros son superiores los valores de la muestra con pianista de danza. Por lo tanto, un mayor tiempo en Esperando Turno pudiera indicar un mayor número de alumnas por clase, de ahí la necesidad de agrupar y secuenciar la variación o ejercicio, pero también de una mayor realización de ejercicios en lo que se llama centro que tradicionalmente es la parte más importante en cualquier clase de técnica de Danza Clásica.

Figura 6 - Frecuencia Esperando Turno (izquierda) y Tiempo Esperando Turno (derecha).

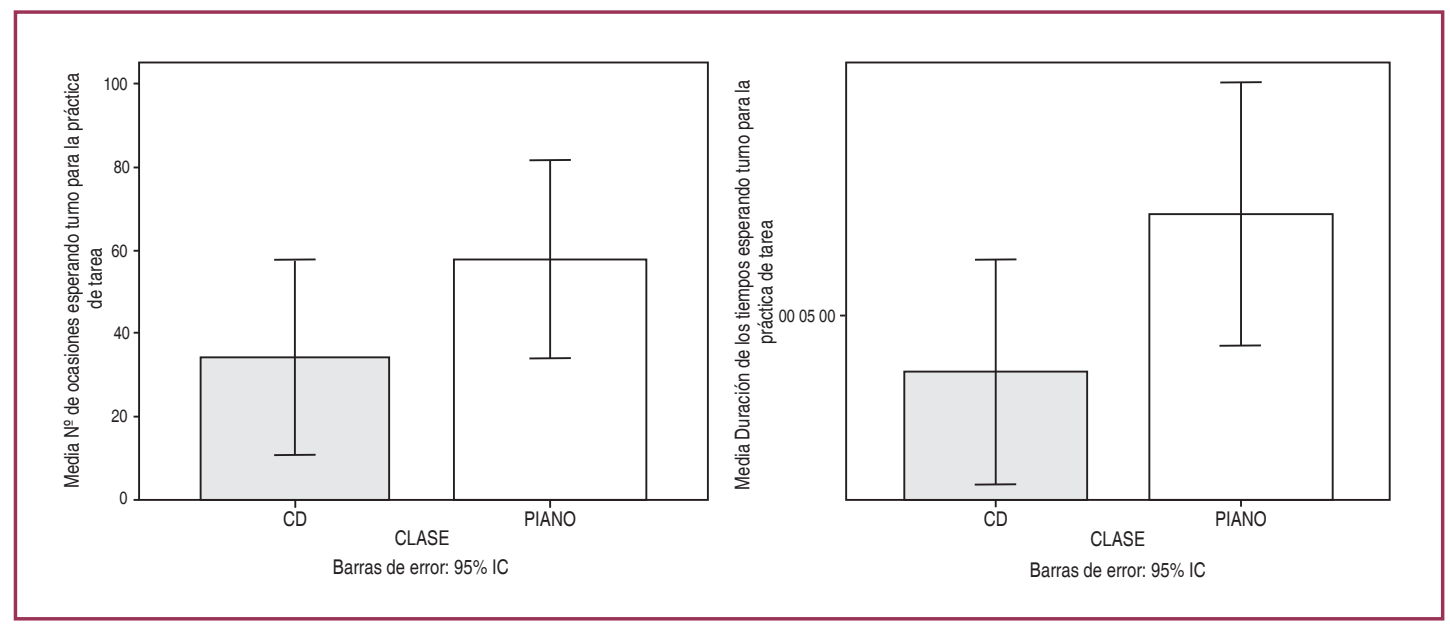




\subsection{OTRAS ACTIVIDADES}

Por último, esta variable recoge imprevistos, interrupciones y toda actividad fuera del orden establecido, es decir, circunstancias que no se corresponden con Explicación, Tiempo de Tarea, o Recibiendo Feedback. No son significativas las diferencias entre ambas muestras, pero sí hay que destacar un menor valor en las ocasiones o frecuencias para la muestra con pianista de danza frente a la muestra con música grabada. En este caso se registraron calentamientos previos al inicio de la clase.

Figura 7 - Frecuencia Otras Actividades (izquierda) y Tiempo Otras Actividades (derecha).

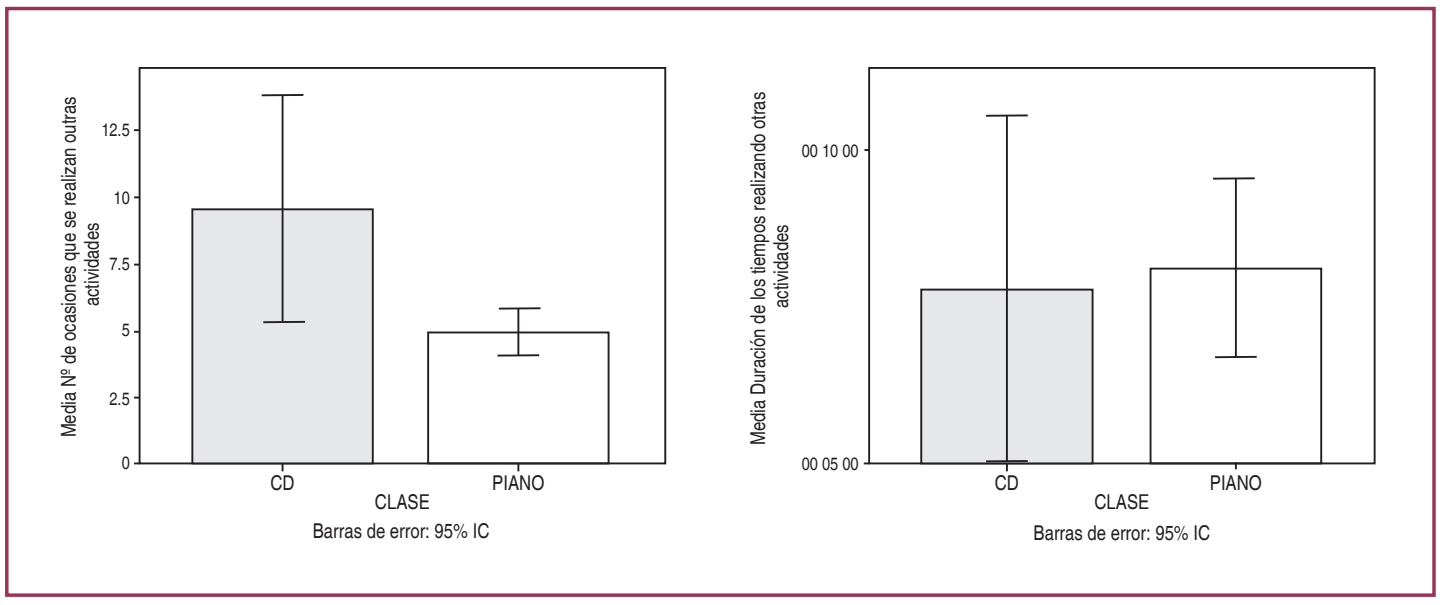

Fuente: Datos de los autores

\section{DISCUSIÓN Y CONCLUSIONES}

A raíz de los datos obtenidos y comentados, puede considerarse que las clases acompañadas mediante el pianista de danza, fueron más fructíferas y provechosas para la consecución de los objetivos educativos y permitieron una menor pérdida de tiempo, frente a las clases con música grabada. Esto se ha apreciado, sobre todo, en las variables Organización y Tiempo de Tarea. En el primer caso, Organización, las diferencias entre ambas muestras fueron hasta cuatro veces más altas en las tareas de organización de la clase en el caso del empleo de la música grabada con respecto al piano. Estos resultados coinciden con los de G-Molero y Romero-Ramos (2016), quienes registraron un total de 3'42" de tiempo total para la búsqueda de la música por parte de la profesora (Tiempo de Organización) en clase con música grabada, frente a 1'2" en la clase con pianista de danza. Por tanto, esta reducción del tiempo organizativo en las sesiones impartidas con pianista, podría propiciar clases más completas, con menor pérdida de tiempo y de esta manera, con mayor tiempo para la ejecución de las variaciones. Como afirman Piéron (1999) y Lozano y Viciana (2003), la correcta gestión del tiempo como competencia docente influye enormemente en el proceso de enseñanzaaprendizaje.

Precisamente, los valores registrados de Tiempo de Tarea indican también valores más altos en las clases acompañadas con piano, lo que supone un incremento de tiempo por clase y, por tanto, en el curso académico. Es necesario recordar aquí como el Tiempo de Tarea o Tiempo de Compromiso Motor, se considera la variable temporal más destacada 
como indicadora del aprendizaje del alumnado (RAMÍREZ; LOZANO; SAN-MATÍAS; ZABALA; VICIANA, 2006, apud BERLINER, 1979; SIEDENTOP, 1983).

Se apreciaron así mismo, diferencias significativas en la frecuencia del marcaje 0 explicación del profesorado en las clases acompañadas con piano: 21,67 $\pm 5,67$ ocasiones, frente a 41,04 $\pm 10,53$ ocasiones, con música grabada. Esto puede indicar menor necesidad de repetición del ejercicio por parte del profesor o bien una mayor comprensión del alumnado ante la variación propuesta.

Los valores que reflejan el turno de práctica, son casi el doble en el caso de las clases con pianista de danza, tanto en la frecuencia, como en el tiempo, mostrándose unas clases más completas con piano, pues los ejercicios de barra preparan los de centro con variaciones grupales más complejas.

Por todo lo visto, dado que las sesiones donde se utilizó el piano registraron valores más altos de Tiempo de Tarea y más bajos en Organización, principalmente, (además de en Explicación y Esperando Turno), se puede concluir que este soporte musical facilitará al profesorado la impartición de las clases previstas, y por tanto, la consecución y logro de los objetivos educativos propuestos, enriqueciendo además la experiencia del alumnado con la música en vivo y facilitando el proceso de enseñanza-aprendizaje. Estos beneficios metodológicos y didácticos se contextualizan en un Conservatorio Superior de Danza que son instituciones públicas que hacen un esfuerzo por ofrecer la mejor calidad de enseñanza posible a sus alumnos, sin embargo somos conscientes que no en todos los lugares se dispone de esta posibilidad pero al menos este trabajo da un soporte científico para poder reclamar en un futuro la presencia de este profesional que permite un mejor aprovechamiento del tiempo de clase y consecuentemente de las experiencias de aprendizaje.

\section{REFERENCIAS}

AGUADO, Saúl. Arreglos de piano para Danza. Madrid: Real Musical, 2009. v. 1.

AGUADO, Saúl. Arreglos de piano para Danza. Madrid: Real Musical, 2009. v. 2.

CAMPOS, Maria del Carmen; GARRIDO, Maria Encarnación; CASTAÑEDA, Carolina. El estilo de enseñanza como determinante del tiempo de compromiso motor en Educación Física.

Scientia: Revista Multidisciplinar de Ciencias de La Salud, v. 16, n. 1, p. 40-51, 2011.

CASAMICHANA, David; CASTELLANO, Julen. Time-motion, heart rate, perceptual and motor behaviour demands in small-sides soccer games: Effects of pitch size. Journal of Sports

Sciences, v. 28, n. 14, p. 1615-1623, 2010.

CARROLL, John. A model for school learning. Teacher College Record, v. 64, p. 723,733, 1963.

CAVALLI, Harriet. Dance and music. Florida: University Press of Florida, 2001.

CAMERINO, Oleguer; CHAVERRI, Javier; ANGUERA, María Teresa; JONSSON, Gudberg. Dynamics of the game in soccer: Detection of t-patterns. European Journal of Sport Science, 
CONSERVATORIO SUPERIOR DE DANZA “ÁNGEL PERICET". Nuestro Centro. Málaga, 2012. Disponible en: http://www.csdanzamalaga.com/nuestro-centro/ Acceso en: 24 nov. 2018.

COTERÓN, Javier; SÁNCHEZ, Galo. La educación artística por el movimiento. La expresión corporal en educación física. Revista de Pedagogía de la Universidad de Salamanca, v.16, p. 113-134, 2010.

DENDENA, Neusa; CARMO, Maria do. Educação Física escolar e dança: percepções de professores no ensino fundamental. Movimento, v. 15, n. 4, p. 193-214, 2009.

FERNANDEZ-REVELLÉS, Andrés. Efecto del conocimiento de resultados de los índices temporales de eficacia sobre la competencia docente Tiempo. 2003. $373 \mathrm{f}$. Tesis (doctoral) - Universidad de Granada, 2003. Disponible en: http://digibug.ugr.es/bitstream/handle/10481/39630/ FernandezRevellés Tiempo .pdf;jsessionid=A3497FE02D45F828960FE5CC8C540AAA?sequence $=1$ Acceso en: 24 nov. 2018.

FERNANDEZ-REVELLÉS, Andrés. El tiempo en la clase de educación física: la competencia docente tiempo. Deporte y Actividad Física para Todos, v. 41, p. 102-120, 2008.

FERNÁNDEZ, Jordi; CAMERINO, Oleguer; ANGUERA, María Teresa; JONSSON, Gudberg K. Identifying and analyzing the construction and effectiveness of offensive plays in basketball by using systematic observation. Behavior Research Methods, v. 41, n. 3, 719-730, 2009.

FERNÁNDEZ, Miguel; SÁNCHEZ, Carmen Rosa; JIMÉNEZ, Francisco; NAVARRO, Vicente; ANGUERA, María Teresa. Sistema de codificación y análisis de la calidad del dato para una intervención inclusiva en Educación Física. Revista de Psicología del Deporte, v. 21, n. 1, p. 67-73, 2012.

FROSI, Simon. The improvisation of structured keyboard accompaniments for the ballet class. 2011. $51 \mathrm{f}$. Tesis (doctoral) - Western Australian Academy of Performing Arts, Edith Cowan University, 2011. Disponible en: http://ro.ecu.edu.au/ theses hons/39 Acceso en: 24 nov. 2018.

G-MOLERO, Hayda; ROMERO-RAMOS, Óscar. Tiempo de compromiso motor según el soporte musical en danza. Revista Iberoamericana de Ciencias de la Actividad Física y el Deporte, v. 5, n. 1, p. 70-94, 2016.

G-MOLERO, Hayda; FERNÁNDEZ-GARCÍA, José Carlos. Observación sistemática de variables temporales e incidencia del soporte musical utilizado en la clase de Danza Clásica. In : CONGRESO INTERNACIONAL DE DANZA, INVESTIGACIÓN Y EDUCACIÓN: DANZA Y COMUNICACIÓN, 6., 2018. [Anales...] Málaga, Universitat de Málaga, 2018. (No prelo)

GARCÍA, Óscar; RIAL, Antonio; REAL, Eulogio. Using heart rate to detect high-intensity efforts during professional soccer competition. Journal of Strength and Conditioning Research, $v$. 26, n. 8, p. 2058-2064, 2012.

LAGUNA, Alejandro César. Revisión de problemas comunicacionales en la clase de técnica de danza observados por un músico de danza. 2013. 134f Tesis (doctoral)- Universidad de Évora, 2013.

GUTIÉRREZ-SANTIAGO, Alfonso; PRIETO, Iván; CAMERINO, Oleguer; ANGUERA, María Teresa. Sequences of errors in the judo throw Morote Seoi Nage and their relationship to the learning process. Journal of Sports Engineering and Technology, v. 227, n. 1, p. 57-63, 2013. 
HERNÁNDEZ, Antonio; CASTELLANO, Julen; CAMERINO, Oleguer; JONSSON, Gudberg; VILLASEÑOR, Ángel; ANGUERA, María Teresa. Programas informáticos de registro, control de calidad del dato, y análisis de datos. Revista de Psicología del Deporte. v. 23, n. 1, p. 111-121, 2014.

HERNÁNDEZ-MENDO, Antonio; MONTORO, Jorge; REINA, Álvaro; FERNÁNDEZ-GARCÍA, José Carlos. Desarrollo y optimización de una herramienta observacional para el bloqueo en voleibol. Revista Iberoamericana de Psicología del Ejercicio y el Deporte, v. 7, n.1, p. 15-32, 2012.

HERRERA, Lucía; GÓMEZ, lleana. Programa de entrenamiento cognitivo-constructivista para piano de acompañamiento de ballet clásico. Publicaciones - Facultad de Educación y Humanidades del Campus de Melilla, v. 41, p. 85-104, 2011.

LEWIS, Sandra; D'ANDREA, Frances Mary; ROSENBLUM, L. Penny. The Development of Accepted Performance Items to Demonstrate Competence in Literary Braille. Journal of Visual Impairment y Blindness, v. 106, n. 4, p. 197-211, 2012.

LÓPEZ, Ana. El pianista acompañante en la clase de danza: problemática actual. Ventajas de la utilización de los recursos creativos. In: CONGRESO EDUCACIÓN E INVESTIGACIÓN MUSICAL (CEIMUS), 1., 2008. [Actas ...].Madrid: Enclave Creativa, 2008.

LÓPEZ, Ana. La improvisación pianística aplicada al acompañamiento de danza. Madrid: CRIF Las Acacias, 2009.

LOSADA, José Luis. Relación entre zonas y contextos de interacción en el fútbol de alto rendimiento: una aplicación multivariante. Motricidad: European Journal of Human Movement, v. 28, p. 171-183, 2012.

LOZANO, Demetrio; CAMERINO, Oleguer. Eficacia de los sistemas ofensivos en balonmano. Apunts. Educación Física y Deportes, v. 108, p. 66-77, 2012.

LOZANO, Luis; VICIANA, Jesús. Las competencias docentes en Educación Física. Un estudio basado en la competencia de gestión del tiempo y la organización de la clase. Investigación en Educación Física y Deportes, v. 1, p. 75-95, 2002.

LOZANO, Luis; VICIANA, Jesús; PIÉRON, Maurice. Análisis de los instrumentos de observación empleados para el registro de variables temporales en educación física. Apunts Educación Física y Deportes, v. 82, p. 22-31, 2006.

METZLER, Michael William. The measurement of academic learning time in physical education. Tesis. The Ohio State University, Columbus, 1979. Disponible en: https://etd. ohiolink.edu/!etd.send file?accession=0su1487090040107823\&disposition=inline Acceso en: 24 nov. 2018.

METZLER, Michael; DEPAEPE, James; REIF, Glenn. Alternative technologies for measuring academic learning time in physical education. Journal of teaching in physical education, $v .4$, n. 4, 271-285, 1985.

MONTÁVEZ, Mar. La expresión corporal en la realidad educativa. Descripción y análisis de su enseñanza como punto de referencia para la mejora de la calidad docente en los centros públicos de educación primaria de la ciudad de Córdoba. 2011. 110f. Tesis (Doctoral) Universidad de Córdoba, Córdoba, España, 2011. 
NEUMUTH, Thomas; KASCHEK, Bertrand; NEUMUTH, Dayana; CESCHIA, Marcello; MEIXENSBERGER, Jürgen; STRAUSS, Gero; BURGERT, Oliver. An observation support system with an adaptive ontology-driven user interface for the modeling of complex behaviors during surgical interventions. Behavior Research Methods, v. 42, n. 4, p. 1049-1058, 2012.

OLMEDO, José Ángel. Estrategias para aumentar el tiempo de práctica motriz en las clases de Educación Física escolar. Apunts: Educación física y deportes, v. 59, p. 22-30, 2000.

PAZ DE, Alberto. La improvisación pianística aplicada al acompañamiento de danza. La Improvisación como sistema pedagógico. Madrid: IEM, 2006.

PAZ DE, Alberto. Acompañamiento pianístico en las clases de ballet. Valladolid: SLKE Campus Virtual, 2011.

PÉREZ, María Elena. Remembranzas de un Pianista. Revista Musical Chilena, n. Esp., p. 7880, 2002.

PÉREZ-CHIRINOS, Christian. La improvisación musical en la clase de danza académica. In: GIMÉNEZ MORTE, Carmen. La Investigación en Danza en España 2014. Valencia: Mahali, 2014. p. 369-378.

PIÉRON, Maurice. Enseñanza de las actividades físicas y deportivas: Observación e investigación. Málaga: Unisport, 1986.

PIÉRON, Maurice. Didáctica de las actividades físicas y deportivas. Madrid: Gymnos, 1988a.

PIÉRON, Maurice. Pedagogía de la actividad física y del deporte. Málaga: Unisport, 1988b.

PIÉRON, Maurice. Para una enseñanza eficaz de las actividades físico-deportivas.

Barcelona: Inde, 1999.

PRIETO, Iván; GUTIÉRREZ-SANTIAGO, Alfonso; CAMERINO, Oleguer; ANGUERA, María Teresa. Knowledge of error in relation to the teaching and learning of the Osoto-Gari Judo throw. International Journal of Sports Science and Coaching, v. 8, n. 1, p. 53-62, 2013.

PUEYO, Carmelo. Música en danza: Manual de música aplicada a la danza académica. Zaragoza: Prames, 2011.

PUEYO, Carmelo Benedicto. Aproximación a la danza: Historia, textos y contextos. versión Kindle, 2016. (Terpsícore n. 2)

RAMÍREZ, Jorge; LOZANO, Luis; SAN-MATÍAS, Julio; ZABALA, Mikel; VICIANA, Jesús. Directrices metodológicas para la observación sistemática del tiempo de clase en la investigación de la educación física. European Journal of Human Movement, v. 15, p. 187196, 2006.

SIEDENTOP, Daryl; BIRDWELL, Deborah; METZLER, Michael. A process approach to measuring teaching effectiveness in physical education. Paper presented at the AAHPERD research symposium: New Orleans, 1979.

SIRERA, Belén; SIRERA, María. El piano en la danza: Investigación musicológica. Madrid: Alpuerto, 2009.

TELAMA, Risto; VARSTALA, Väinö; HEIKINARO-JOHANSSON, Pilvikki; PAUKKU, Paul. The relationship between pupil's leisure time physical activity and motor behavior during physical 
education lessons. In: PIÉRON. M.; GRAHAM, G. Sport Pedagogy. Champaign: Human Kinetics, p. 57-62, 1986.

TELLO, Isaac. El acompañamiento pianístico de la danza: la improvisación como recurso creativo. Tesis doctoral, Facultad de Educación de la Universidad Complutense de Madrid, Madrid, España, 2016. Disponible en: https://eprints.ucm.es/40384/1/T38087.pdf Acceso en: 24 nov. 2018.

TROYA, Yanira; CUÉLLAR, María Jesús. Formación docente y tratamiento de la danza en Canarias: evaluación desde la Educación Física. Retos. Nuevas tendencias en Educación Física, Deporte y Recreación, v. 24, p. 165-170, 2013.

VALLÉS GRAU, Luis. La especialidad de pianista acompañante en la titulación superior de música: una propuesta de currículum e integración en el sistema educativo español. Tesis doctoral. Universidad Jaume I, Castellón, España, 2015. Disponible en: <http://hdl.handle. net/10803/319458> Acceso en: 24 de noviembre de 2018.

WONG, Yee Sik. The art of accompanying classical ballet technique classes. Tesis doctoral. University of lowa. lowa, Estados Unidos, 2011. Disponible en: <http://ir.uiowa.edu/etd/1192/> Acceso en: 24 de noviembre de 2018.

YANCI, Javier; VINUESA, Anabel; RODRÍGUEZ-NEGRO, Josune; YANCI, Leire. El tiempo de compromiso motor en las sesiones de Educación Física del primer y segundo ciclo de Educación Primaria. Sportis. Scientific Technical Journal of School Sport, Physical Education and Psychomotricity, v. 2, n. 2, p. 239-253, 2016.

\section{Agradecimientos:}

Quisiéramos agradecer la colaboración prestada por el Conservatorio Superior de Danza de Málaga, donde se realizó la investigación, a su director, profesores de Danza Clásica, profesores pianistas acompañantes y al alumnado participante, así como a Javier Yanci Irigoyen que nos facilitó el software MOTS y que hicieron posible la realización de este trabajo de investigación. 